$\mathrm{J} 3 \mathrm{eA}$, Journal sur l'enseignement des sciences et technologies de l'information et des systèmes, Volume 4, Hors-Série 2, 34 (2005)

DOI : http://dx.doi.org/10.1051/bib-j3ea:2005734

(C) EDP Sciences, 2005

\title{
Étude expérimentale d'une liaison par fibre optique
}

\author{
E. Cassan
}

\author{
Institut d'Électronique Fondamentale \\ Université Paris-Sud Orsay \\ Bâtiment 220
}

F-91405 Orsay CEDEX, France 


\title{
ETUDE EXPERIMENTALE D'UNE LIAISON PAR FIBRE OPTIQUE
}

\author{
Eric Cassan, Institut d'Electronique Fondamentale, Université Paris-Sud Orsay, Bâtiment \\ 220, 91405 Orsay Cedex, eric.cassan@ief.u-psud.fr
}

\section{Résumé :}

Le secteur des télécommunications s'est considérablement développé depuis quelques années. Dans ce contexte, les télécommunications optiques occupent une place de choix pour le transport des données sur des distances dépassant quelques $\mathrm{km}$. La combinaison de sources lasers rapides émettant dans le proche infra-rouge (principalement autour de $\lambda=1,55 \mu \mathrm{m}$ ), d'amplificateurs optiques, et les qualités propres de la fibre optique (pertes faibles : $\approx 0,2 \mathrm{~dB} / \mathrm{km}$ vers $\lambda=1,55 \mu \mathrm{m}$, et dispersion chromatique acceptable), ont rendu cette évolution possible. A ceci s'ajoute la possibilité de "combiner" les signaux optiques véhiculés par la fibre via la technique du multiplexage en longueur d'onde (Wavelength Division Multiplexing ou WDM), qui permet d'atteindre des débits binaires de quelques Térabits par seconde transportés par une unique fibre. Il est donc important de sensibiliser les étudiants EEA aux problèmes spécifiques aux transmissions par fibre optique.

Nous présentons dans cette communication une expérience menée dans ce sens à Orsay, dans le cadre de la maîtrise EEA et du DESS Systèmes Electroniques, qui est basée sur l'exploitation d'une liaison point à point mono-longueur d'onde.

Mots clés : Fibre optique, diode laser, photodiode, lentilles minces, pertes de propagation, ouverture numérique.

\section{DESCRIPTION DE LA MANIPULATION}

La liaison proposée est constituée

- d'une diode laser Fabry-Perot émettant dans le visible autour de $\lambda=645 \mathrm{~nm}$, et pouvant être alimentée en courant continu ou modulé (modulation sinusoïdale),

- de plusieurs sections de fibres optiques que l'on peut mettre en série via des connecteurs (fibre multimode à gradient d'indice $50 \mu \mathrm{m} / 125 \mu \mathrm{m}$ ),

- d'un photodétecteur de type photodiode PIN silicium polarisée en inverse sous $12 \mathrm{~V}$ (voir Fig. 1).

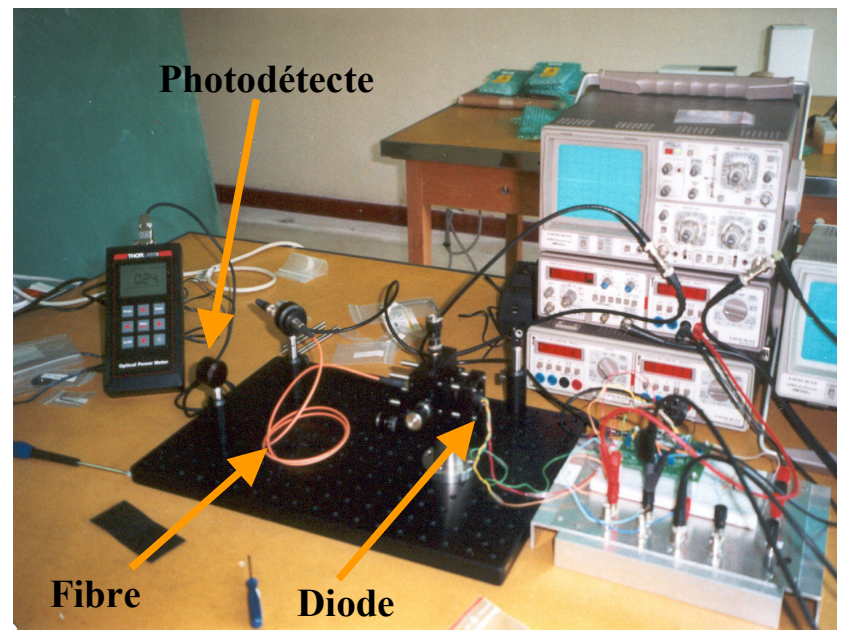

fig. 1 : Vue d'ensemble d'une partie de la manipulation

Un dispositif est prévu pour coupler au mieux le faisceau émis par la diode laser dans la fibre optique (voir Fig. 2). Ce faisceau traverse une lentille convergente de collimation de faible distance focale $(4,5 \mathrm{~mm})$, notée $\left(\mathrm{L}_{1}\right)$, qui permet d'obtenir un faisceau de rayons parallèles. Une deuxième lentille, dite de focalisation (lentille $\left(\mathrm{L}_{2}\right)$ ), a pour but de produire une tâche optique de dimension compatible avec le diamètre de cœur de la fibre $(50 \mu \mathrm{m})$. Cette deuxième lentille convergente possède une distance focale assez grande $(11 \mathrm{~mm})$ pour qu'une fraction importante de la lumière attaque la face d'entrée de la fibre à incidence suffisamment rasante pour appartenir au cône d'acceptance de la fibre définie par son ouverture numérique $(\mathrm{ON}=0,22)$. En sortie, la lumière issue dans la fibre illumine la partie photo-sensible du photodétecteur à entrée fibrée, dont on mesure le photocourant débité sur une impédance de charge ( $1 \mathrm{M} \Omega$, ou $1 \mathrm{k} \Omega$, ou $50 \Omega$ ). Tous ces éléments sont montés sur un ensemble de couplage opto-mécanique possédant les 3 degrés de liberté mécaniques nécessaires pour assurer un positionnement correct du faisceau focalisé sur la face d'entrée de la fibre. La précision de ce positionnement est cruciale, et se chiffre à quelques dizaines de microns au plus. Les étudiants disposent en outre d'un puissancemètre et de lunettes de protection laser adaptées à la longueur d'onde de travail.

\section{CONTENU PEDAGOGIQUE}

En-dehors même du détail des grandeurs physiques mesurées et des phénomènes mis en évidence, ce type de manipulation sur banc optique permet en premier lieu de sensibiliser les étudiants EEA aux aspects liés à la sécurité laser et à la spécificité des manipulations sur banc optique. D'une part, le 


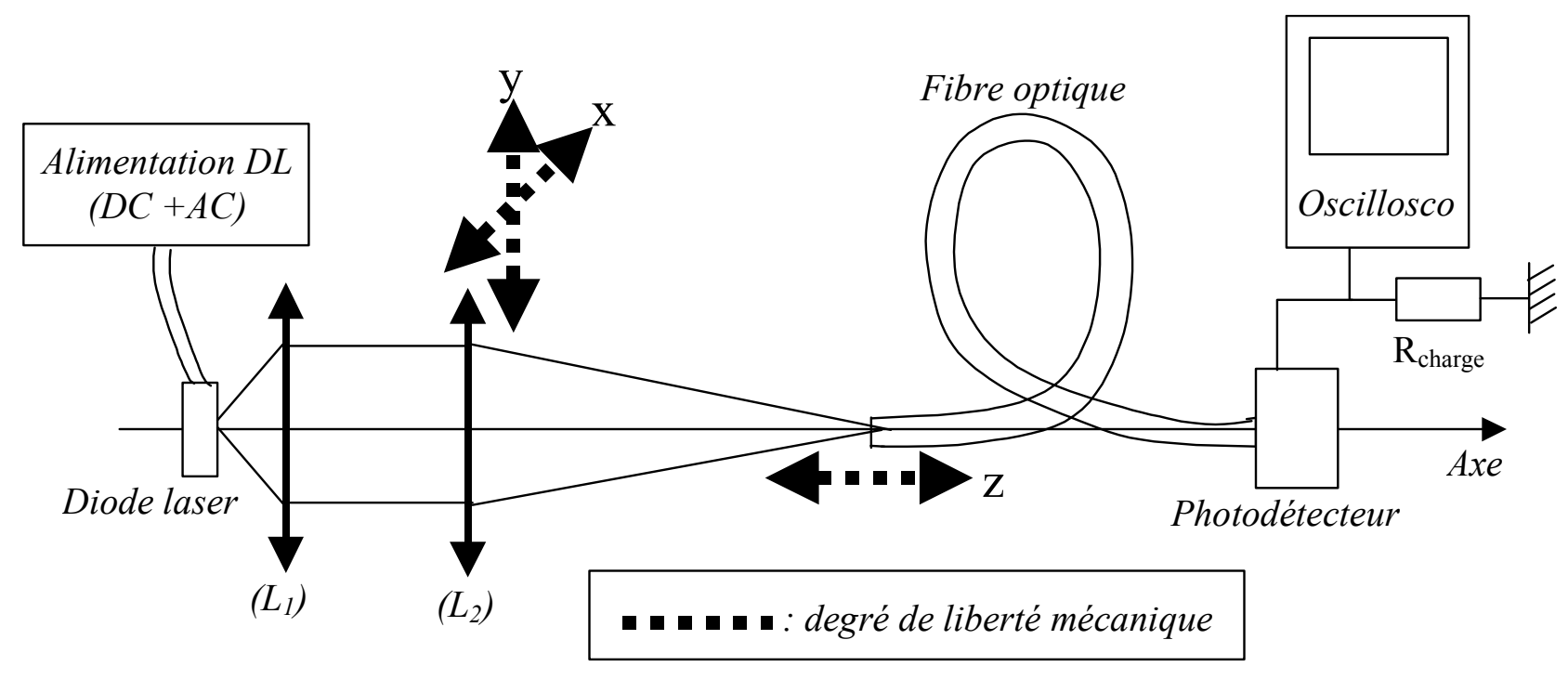

fig. 2 : Schéma de principe de la manipulation

matériel est coûteux (une paire de lunettes de protection coûte environ $200 €$ ) et fragile. Il est donc demandé aux étudiants de manipuler les connecteurs avec soin, de ne pas rayer les lentilles, etc .... . D'autre part, c'est également l'occasion pour eux d'appréhender la sensibilité des réglages optomécaniques propres à l'optique. A ce titre, le réglage proposé aux étudiants doit être mené avec soin.

\subsection{Caractéristiques externes de la diode laser}

- Le TP commence par une évaluation du courant de seuil de la diode laser, une assez forte dispersion existant en effet entre les différentes diodes d'un même lot $\left(\mathrm{I}_{\text {seuil }}\right.$ de 25 à $\left.60 \mathrm{~mA}\right)$. Les pentes au-dessus du seuil $\Delta \mathrm{P} / \Delta \mathrm{I}$ mesurées expérimentalement sont de l'ordre de $0,4 \mathrm{~mW} / \mathrm{mA}$. Le rendement externe est donc de l'ordre de $20 \%$.

- La deuxième étape proposée consiste ensuite à obtenir un faisceau collimaté dont la taille est compatible avec la surface photosensible du puissancemètre. La lentille $\mathrm{L}_{1}$ est montée seule sur le support XYZ, et l'on observe à faible puissance la tâche lumineuse en champ lointain (il s'agit de placer la diode laser au plan focal objet de la lentille).

- Après fixation de $\mathrm{L}_{1}$, on procède ensuite au tracé

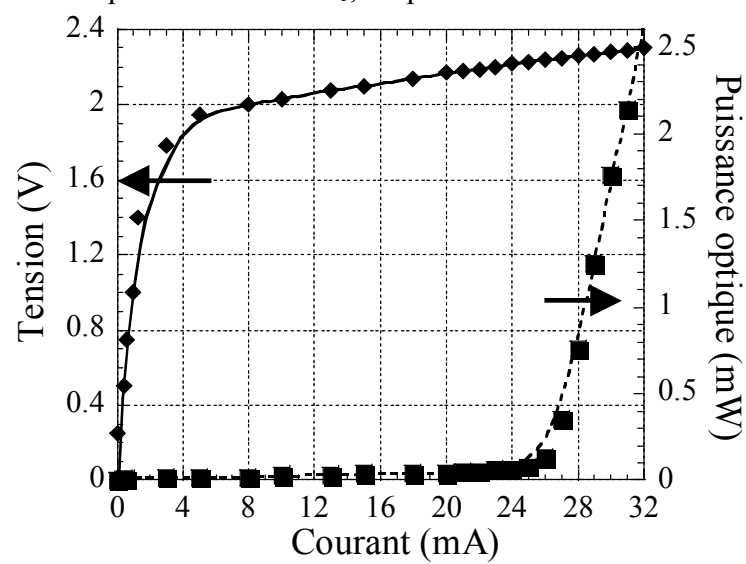

fig. 3 : Caractéristiques typiques d'une DL utilisée des caractéristiques $\mathrm{I}(\mathrm{V})$ et $\mathrm{P}(\mathrm{I})$ de la diode laser à disposition (cf fig. 3).

\subsection{Couplage de la lumière émise par la diode laser dans la fibre optique}

- Après vérification de l'alignement du faisceau collimaté avec l'axe optique sur lequel la fibre optique va être montée, on place la lentille de focalisation $\mathrm{L}_{2}$.

- Le couplage de la lumière dans la fibre optique multimode est obtenu en agissant sur les 3 degrés de liberté disponibles (la fibre est mobile en translation sur l'axe optique, et la lentille $\mathrm{L}_{2}$ selon les deux autres directions). La section de fibre de $1 \mathrm{~m}$ utilisée est connectée à la photodiode PIN silicium, qui fournit un signal électrique permettant l'optimisation du couplage.

- Dans un premier temps, tout le problème est de détecter un quelconque signal. Il s'agit ensuite d'optimiser de proche en proche la photo-réponse. Quand le réglage est jugé optimum, on fixe les différents éléments mécaniques. Connaissant la sensibilité du détecteur à la longueur d'onde de $645 \mathrm{~nm}(0,45 \mathrm{~A} / \mathrm{W})$ et la résistance de charge $\mathrm{R}_{\text {charge}}$, la mesure de la photo-tension $\mathrm{V}_{\mathrm{ph}}$ aux bornes de $\mathrm{R}_{\text {charge }}$ permet d'évaluer la puissance optique couplée dans la fibre et donc le rendement de couplage. Selon les diodes lasers utilisées et la finesse des réglages, des rendements de couplage de 30 à $80 \%$ sont obtenus expérimentalement. Les diodes laser délivrant typiquement $1 \mathrm{~mW}$ pour un niveau de courant supérieur au courant de seuil de quelques $\mathrm{mA}$, des niveaux de puissance importants de quelques centaines de $\mu \mathrm{W}$ sont disponibles en détection.

- Connaissant les valeurs extrêmes des deux angles $\theta_{/ /}$et $\theta_{\perp}$ du diagramme d'émission des diodes lasers (voir fig. 4), on demande aux étudiants de chiffrer 
l'ordre de grandeur théorique des rendements de couplages diode $\rightarrow$ fibre. Ces estimations conduisent à une plage d'efficacité de couplage allant de $40 \%$ à $100 \%$, en accord correct avec les résultats des manipulations.

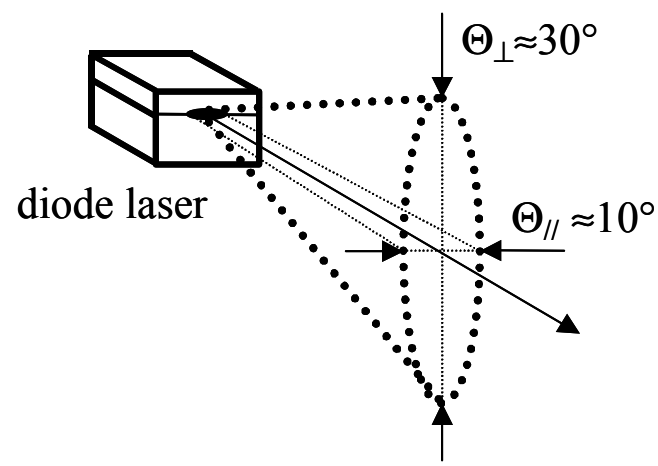

fig. 4 : Diagramme d'émission typique d'une diode laser à émission par la tranche

\subsection{Caractéristiques de la fibre optique}

- Le niveau de puissance disponible permet l'observation de la dimension W de la tâche lumineuse dans un plan situé à une distance L. Cette méthode, bien que peu précise, permet une évaluation de l'ouverture numérique de la fibre (cf fig. 5). Les étudiants mesurent une ouverture $\mathrm{ON}$ de l'ordre de 0,2 , en bon accord avec les indications du constructeur $(\mathrm{ON}=0,22)$.

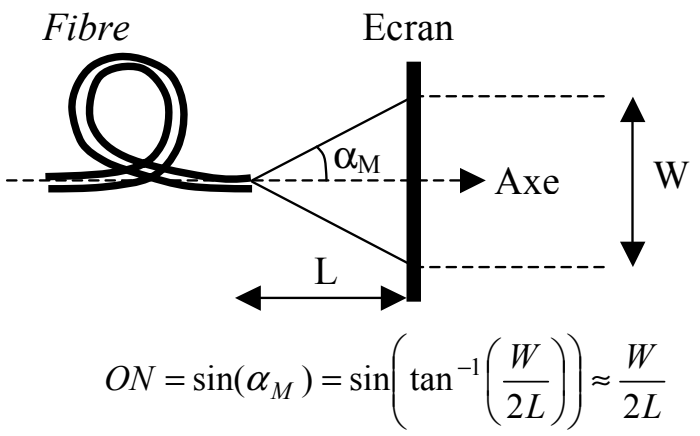

fig. 5 : Méthode approchée de mesure de l'ON de la fibre

- Tous les éléments, depuis la diode laser jusqu'à la section de fibre de $1 \mathrm{~m}$ ayant été fixés avec soin, les pertes de propagation sont ensuite évaluées en relevant les photo-tensions $\left(\mathrm{V}_{\mathrm{ph} 1}, \mathrm{~V}_{\mathrm{ph} 2}, \mathrm{~V}_{\mathrm{ph} 3}\right)$ dans les 3 cas suivants :

(1) $\mathrm{L}_{1}=1 \mathrm{~m}$ de fibre

(2) $\mathrm{L}_{1}=1 \mathrm{~m}$ de fibre, 1 connecteur, $\mathrm{L}_{1}=1 \mathrm{~m}$ de fibre

(3) $\mathrm{L}_{1}=1 \mathrm{~m}$ de fibre, 1 connecteur (le même), $\mathrm{L}_{2}=500 \mathrm{~m}$ de fibre
Sans (nécessairement) connaître le niveau de puissance optique injectée dans la fibre $\left(\mathrm{P}_{\text {inj }}\right)$, on en déduit les pertes $\alpha_{\mathrm{CON}}(\mathrm{dB})$ du connecteur et les pertes de propagation $\mathrm{A}(\mathrm{dB} / \mathrm{km})$ de la fibre à la longueur d'onde de $645 \mathrm{~nm}$ :

$\mathrm{P}_{1 \mathrm{dBm}}=\mathrm{P}_{\text {injdBm }}-\mathrm{A} \times \mathrm{L}_{1}$

$\mathrm{P}_{2 \mathrm{dBm}}=\mathrm{P}_{\text {injdBm }}-2 \times \mathrm{A} \times \mathrm{L}_{1}-\alpha_{\mathrm{CON}}$

$\mathrm{P}_{3 \mathrm{dBm}}=\mathrm{P}_{\text {injdBm }}-\mathrm{A} \times\left(\mathrm{L}_{1}+\mathrm{L}_{2}\right)-\alpha_{\mathrm{CON}}$

$$
\begin{gathered}
\alpha_{C O N}=\left(P_{1 d B m}-P_{2 d B m}\right)-A \times L_{1}=10 \lg \left(\frac{V_{p h 1}}{V_{p h 2}}\right)-A \times L_{1} \\
A=\frac{P_{2 d B m}-P_{3 d B m}}{L_{2}-L_{1}}=\frac{10 \lg \left(\frac{V_{p h 2}}{V_{p h 3}}\right)}{L_{2}-L_{1}}
\end{gathered}
$$

Ces mesures donnent des pertes de propagation de l'ordre de 8 à $10 \mathrm{~dB} / \mathrm{km}$, en bon accord avec la littérature à $\lambda=645 \mathrm{~nm}$, et des pertes de connecteur de 1 'ordre de $0,5 \mathrm{~dB}$ à $1 \mathrm{~dB}$.

\subsection{Liaison analogique}

On superpose ensuite un courant variable (sinusoïdal) au courant de repos de la diode laser, lui-même choisi au-dessus du seuil, et l'on qualifie globalement la bande passante de la liaison analogique ainsi constituée en observant la photoréponse à l'oscilloscope. Les étudiants peuvent observer que même dans le cas d'une section de fibre de $500 \mathrm{~m}$, la bande passante de la liaison est limitée par le générateur disponible, dont la fréquence limite est de $20 \mathrm{MHz}$.

\section{CONCLUSION}

Cette expérience pédagogique est menée en complément de TP de simulation des systèmes de communications par fibre optique. Elle a été bien ressentie par les étudiants, pour qui elle a permis d'appréhender d'un point de vue pratique certains aspects des liaisons par fibre et des lasers à semiconducteurs. Nous prévoyons dans l'année à venir d'enrichir le canevas proposé au travers d'une collaboration avec une équipe de recherche de l'Institut d'Electronique Fondamentale (UMR CNRS 8622 , Orsay).

\section{Bibliographie}

1. Harry J. R. Dutton, "Understanding Optical Communications", presses IBM, première édition (septembre 1998).

2. Michel et Irène Joindot, «Télécommunications par fibres optiques », Dunod (1996).

3. P. Lecoy, "Télécoms sur fibres optiques", Hermes (réseaux et télécommunications), deuxième édition (1997). 